\title{
Randomized Controlled Trial of Neurologic Music Therapy in Parkinson's Disease: Research Rehabilitation Protocols for Mechanistic and Clinical Investigations
}

Isabelle Buard ( $\nabla$ Isabelle.Buard@cuanschutz.edu )

University of Colorado Denver https://orcid.org/0000-0001-9455-6365

\section{Lucas Lattanzio}

University of Colorado Denver - Anschutz Medical Campus: University of Colorado - Anschutz Medical

Campus

Rebekah Stewart

Rehabilitative Rhythms

Sarah Thompson

Rehabilitative Rhythms

Kristin Sjoberg

Rehabilitative Rhythms

Karen Hookstadt

University of Colorado Health

Meghan Morrow

University of Colorado Health

Samantha K Holden

University of Colorado Denver - Anschutz Medical Center

Stefan Sillau

University of Colorado at Denver and Health Sciences Center: University of Colorado - Anschutz Medical

Campus

Michael Thaut

University of Toronto

Benzi Kluger

University of Rochester Department of Neurology

\section{Study protocol}

Keywords: Magnetoencephalography, motor cortical activity, neuronal entrainment, rehabilitation, Parkinson's disease, Neurologic Music Therapy 
Posted Date: May 11th, 2021

DOl: https://doi.org/10.21203/rs.3.rs-506372/v1

License: (c) (i) This work is licensed under a Creative Commons Attribution 4.0 International License. Read Full License 


\section{Abstract}

\section{Background}

Presently available medications and surgical treatments for Parkinson's disease have limited effects on fine motor problems and often leave patients with significant fine motor disability. Standard of care occupational therapy (OT) yields low efficacy, potentially due to a lack of standard protocols. Neurologic Music Therapy (NMT) techniques, especially Rhythmic Auditory Stimulation which relies on interaction between rhythm and movement, have shown to be effective in PD gait rehabilitation possibly through their reliance on neural pathways that are not affected by PD. Therapeutic Instrumental Music Performance (TIMP) is one other NMT technique that holds promise but which mode of action and efficacy has not been investigated in PD yet.

Methods

One hundred PD participants will be randomly assigned to receive 15 sessions of either TIMP with rhythm or TIMP without rhythm, standard of care OT, or to be waitlisted (control) over 5 consecutive weeks. Brain oscillatory responses will be collected using magnetoencephalography during an auditory-motor task to understand the underlying mechanisms. The Grooved Pegboard, the UPDRS III finger tap and the fingerthumb opposition will be assessed to investigate clinical changes related to fine motor function. This project will also serve to confirm or refute our pilot data findings suggesting NMT relies on compensatory brain networks utilized by the PD brain to bypass the dysfunctional basal ganglia.

Discussion

This study aims to use standardized TIMP and OT research protocols for investigating the neuronal pathways utilized by each intervention and possibly study their efficacy with respect to fine motor rehabilitation via a randomized control trial in the PD population.

\section{Full Text}

Due to technical limitations, full-text HTML conversion of this manuscript could not be completed. However, the manuscript can be downloaded and accessed as a PDF.

\section{Figures}




\section{Enrollment}

\begin{tabular}{|l|l|}
\hline \multicolumn{2}{|l|}{ Assessed for eligibility } \\
\cline { 2 - 3 }
\end{tabular}$\quad$\begin{tabular}{ll}
\hline \\
\hline
\end{tabular}$\quad$\begin{tabular}{ll} 
Excluded \\
- & Do not meet inclusion criteria \\
- & Other reasons \\
\hline
\end{tabular}

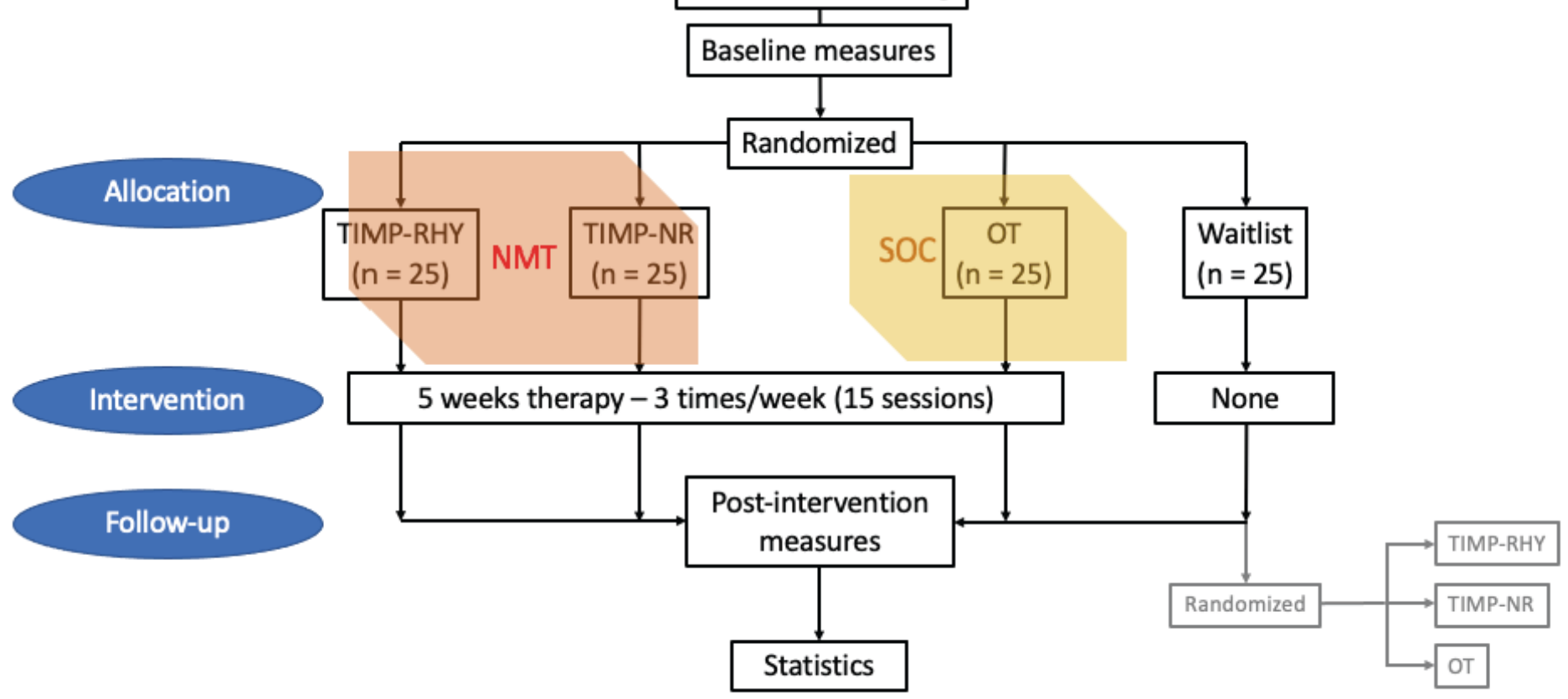

Figure 1

Study Flow 